\title{
Probiotics: current landscape and future horizons
}

\author{
Richard LJ Day ${ }^{\ddagger 1}$, Ashton J Harper ${ }^{\ddagger, 1}$, Rachel M Woods²， Owen G Davies² \& Liam M \\ Heaney*,2(1) \\ ${ }^{1}$ ADM Protexin Ltd, Lopen Head, Somerset, TA13 5JH, UK \\ ${ }^{2}$ School of Sport, Exercise \& Health Sciences, Loughborough University, Loughborough, LE11 3TU, UK \\ *Author for correspondence: I.m.heaney2@|boro.ac.uk \\ $¥$ Authors contributed equally
}
" probiotic strains should be selected for products based on evidence of their phenotype rather than simply relying on the popularity of their species name."

In recent years there has been a rapid rise in interest for the application of probiotic supplements to act as mediators in health and disease. This appeal is predominantly due to ever-increasing evidence of the interaction of the microbiota and pathophysiological processes of disease within the human host. This narrative review considers the current landscape of the probiotic industry and its research, and discusses current pitfalls in the lack of translation from laboratory science to clinical application. Future considerations into how industry and academia must adapt probiotic research to maximize success are suggested, including more targeted application of probiotic strains dependent on individual capabilities as well as application of multiple advanced analytical technologies to further understand and accelerate microbiome science.

Lay abstract: The global market for probiotic supplements is continually expanding. Despite the public perception of benefits provided by probiotics, the evidence to conclusively link probiotic strains to improved characteristics of health or disease is lacking. This is owing, in part, to the lack of large-scale research trials, but also to the insufficient understanding of the interactions occurring within the human system following supplementation. More in-depth research into individual probiotic strains, combined with the application of multiple advanced measurement techniques will provide a future direction for probiotic research and, in turn, aim to provide useful data to translate into routine healthcare practice.

First draft submitted: 11 January 2019; Accepted for publication: 20 March 2019; Published online: 3 May 2019

Keywords: disease $\bullet$ health $\bullet$ metabolomics $\bullet$ metagenomics $\bullet$ metatranscriptomics $\bullet$ microbiome $\bullet$ probiotics $\bullet$ proteomics

The symbiotic relationship between the human host and its bacterial residents has gained extensive research interest in recent years. These bacteria are predominately present within the gastrointestinal system, and along with their collective genomes, are known as the gut microbiome [1]. Increasing evidence suggests that the gut microbiome plays an important role in human host homeostasis, health and disease (Figure 1). Positive effects of gut bacteria have been largely associated with gastrointestinal function, appetite and immune response [2-5]. However, a number of negative effects have been reported across a wide-range of diseases and health conditions, with many associations based on a shift in gut bacterial content and the interaction of metabolites produced following bacterial metabolism of dietary components [6-13]. For these reasons, there has been an invested interest in the application of probiotic supplements as both potential promoters of good health, together with therapeutic modulators of disease development and progression. This narrative review article looks to address the current knowledge and landscape of probiotic research and provide a brief overview to existing applications in health and disease, as well as discuss the potential future directions of probiotic investigations and subsequent translation to the global industry and market. 


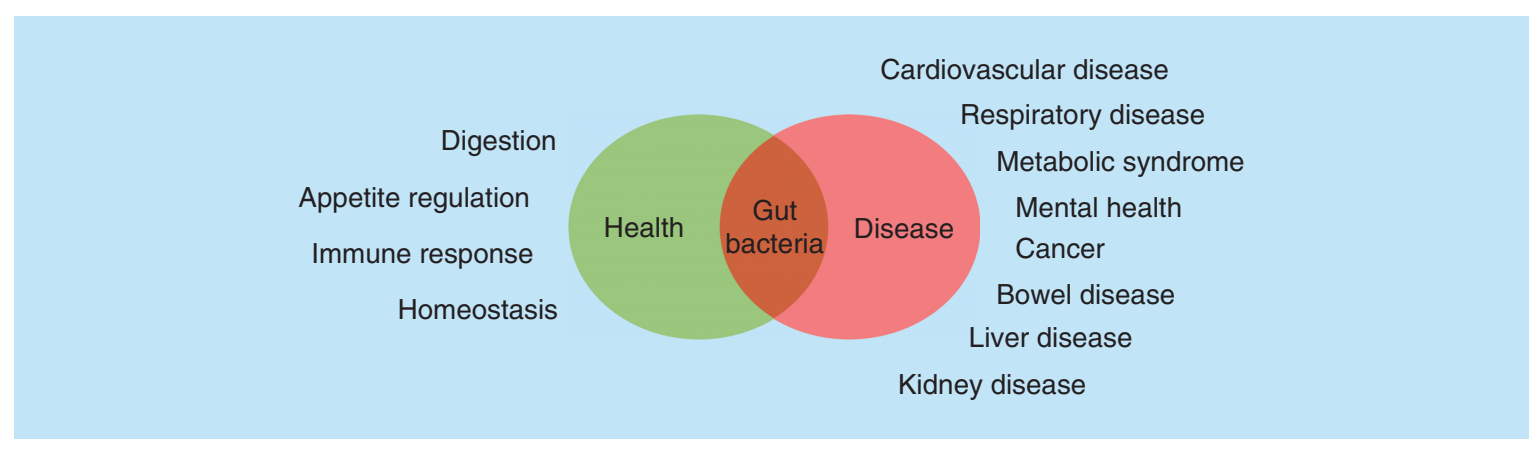

Figure 1. A diagrammatic overview of the positive and negative influence of gut bacteria represented across a wide-range of health and disease conditions.

\section{Lots of research, but still no health claim}

The global probiotic market is worth approximately $\$ 15$ billion USD per year and is growing at an estimated annual rate of 7\% [14]. In recent years, there has been a flourish of interest in research in the microbiome and probiotics. A literature search for probiotics carried out today will return over 19,000 results including some of the most widely read and reputable scientific publications. In particular, a MEDLINE/PubMed search for literature that matches the terms 'probiotics' alongside 'health' OR 'disease' will list almost 10,000 results and demonstrates a rapid rise in research publications with an approximate threefold annual increase in the previous decade and 34-fold increase since 1998 (Figure 2). These include clinical trials, reviews and meta-analyses focusing on clinical presentations as diverse as atopic dermatitis to Clostridium difficile-associated diarrhea (CDAD) [15-17], to anxiety and depression [18-20]. Nevertheless, this extensive body of clinical research has not translated into clearly defined health or medicinal claims for probiotics.

Despite mounting evidence for the use of probiotics in a range of conditions, relatively few strains are commercially available and probiotics are seldom used in routine clinical practice [14]. The European Food Safety Authority (EFSA) has listed three main regulatory issues to be addressed when making a health claim. This has led to many health claims for probiotics being rejected to date, and has even restricted the use of the term 'probiotic' as it is believed that the name itself implies a health benefit. These regulatory issues include the requirement for the demonstrated evidence of:

- Characterization of the probiotic product;

- Substantiation of the health benefit (i.e., demonstration that the biomarker in question contributes to the claimed health benefit), and;

- Extrapolation to the general, healthy population.

Health claims must be specific and claims such as 'strengthens the immune system' are considered too vague by EFSA [21]. Much research has gone into establishing an evidence base for probiotics having an impact on various biomarkers. However, there is no established set of approved biomarkers which are correlated with (and validated by) clinical endpoints [22]. EFSA requires claimed health effects to be demonstrated in at least two randomized, placebo-controlled clinical trials, with a clearly demonstrated cause and effect relationship. It has been suggested that poor study design, a lack of investment in research and development, and a lack of fundamental knowledge about the biological mechanisms of action has led to 'pilotitis' - whereby small and diffused projects do not create a sufficient evidence-base to obtain regulatory approval [14]. A clear example of this is found in a recent systematic review of randomized controlled trials (RCTs) investigating probiotics in the management of irritable bowel syndrome (IBS). From the 35 RCTs reviewed, of which only three had at least 100 participants, the authors concluded that approximately $75 \%$ were pilot studies [23]. Adequately powered Phase III probiotic clinical trials are required to provide the level of evidence that will generate confidence in product efficacy to clinicians and health authorities. 

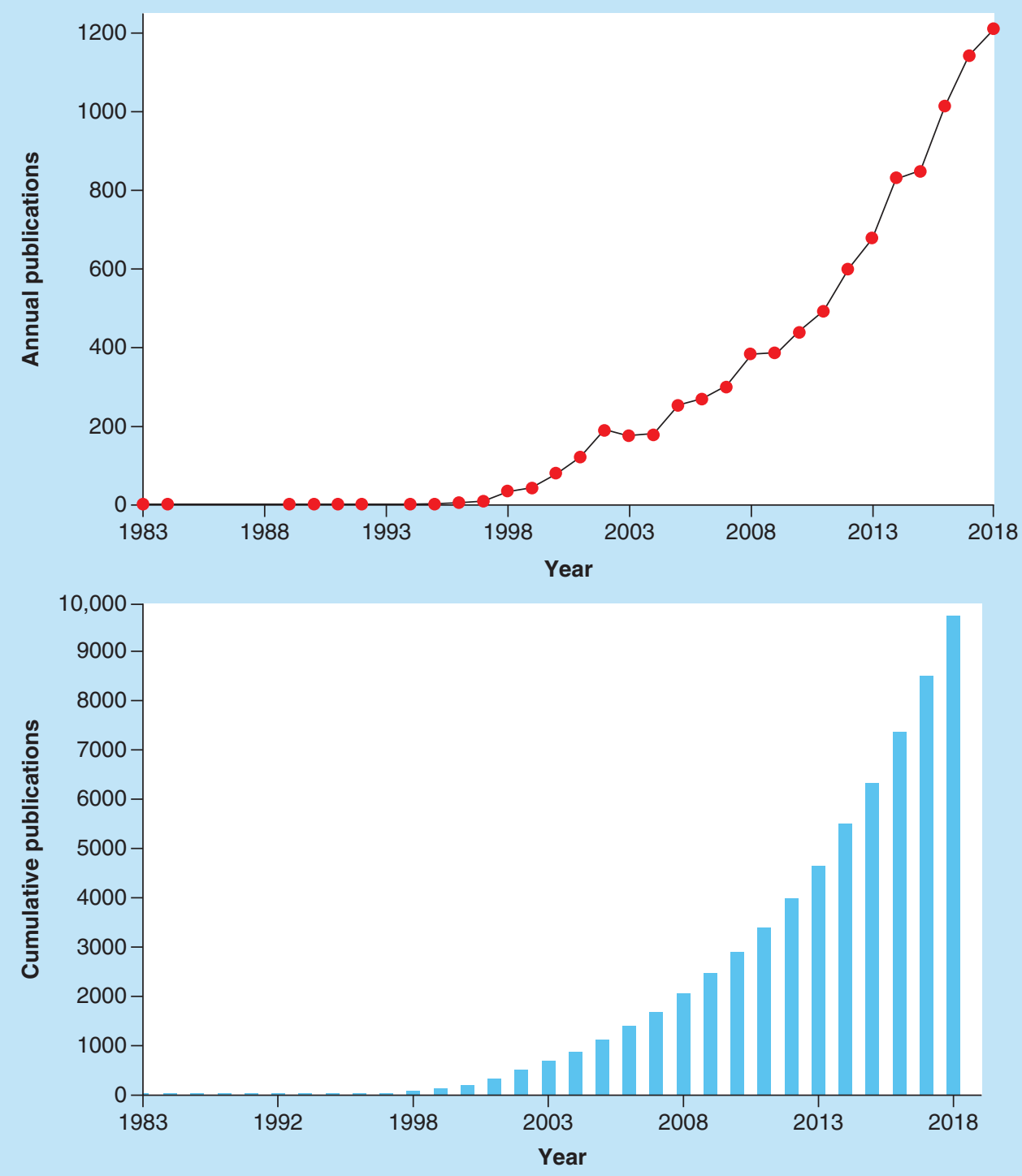

Figure 2. Annual (top) and cumulative (bottom) trend of published research manuscripts indexed on MEDLINE/PubMed matching the terms 'probiotics' AND ('health' OR 'disease') [data refer to search conducted on 27 November 2018].

\section{Diversity, dysbiosis \& strain specificity}

The Human Microbiome Project (HMP1), a National Institute of Health-funded project based on 250 healthy volunteers, discovered that the human microbiome is comprised of between 3500 and 35,000 species; inferred from operational taxonomic units [24]. The total number of bacterial cells present within the human body has been estimated at $10^{13}$, a value similar to that of the number of host cells [25]. The human body thus accommodates a very diverse and abundant collection of microorganisms; however, there is considerable variability in diversity between body sites. For example, oral and colonic sites have bacteria highest in quantity, with lowest abundances measured in the vagina [24]. High gastrointestinal microbial diversity is found in hunter-gatherer populations, such as the Hadza in Tanzania [26] and the Yanomami in Brazil [27], which likely represent our ancestral human state. These dwindling cultural groups show evidence of good health in contrast to modern Westernized populations colonized by a far less diverse microbiota [28]. Low diversity in the lower GI tract (GIT) is associated with a range of conditions including obesity, Crohn's disease, IBS and colorectal cancer [29]. However, high diversity is not a 
universal marker of a healthy microbiome, given that such a state in the vagina, for instance, defines bacterial vaginosis [30]; the most common cause of vaginitis.

Diversity frequently appears in definitions of dysbiosis; a commonly used term in this field referring to an 'abnormal' microbiome. Dysbiosis, which is frequently ill-defined in many publications, appears to cover not only changes in diversity, but also loss in keystone taxa (important species that drive community composition and function), proliferation of pathogens, and changes in metabolic capacity; the latter adding a functional component to the former structural elements [31]. Dysbiosis is not easily defined owing to the fact that eubiosis, a healthy or homeostatic microbiome, is such a heterogeneous state [32]. Microbiome profiling studies have identified unique interpersonal taxonomical patterns, even among twins [33], however the existence of a stable core of functions and thus genes (the microbiome core) has been substantiated [32]. What is yet to be clearly defined is what constitutes a 'healthy' gut microbiota and exactly how different strains and species of gut bacteria affect their host. Interstudy variations in strains, doses and treatment duration make comparing studies and reaching a general consensus difficult.

The genetic difference between one probiotic bacterium and the next may be larger than the difference between a man and a goldfish [34]; as such the function of one strain cannot always be extrapolated to another. This is referred to as strain specificity, and is clearly demonstrated in the study by Douillard et al. [35] in which 100 strains of the species Lactobacillus rhamnosus, obtained from both human and dairy sources, display very different characteristics such as bile acid resistance, carbohydrate transport and metabolism, and production of mucus-binding pili. This study lends weight to the fact that taxonomic profiling is an inadequate measure of functional capacity, and that probiotic strains should be selected for products based on evidence of their phenotype rather than simply relying on the popularity of their species name.

\section{Multi-omics technologies to revolutionize \& accelerate microbiome science}

The microbiome and its influence on the human host is not solely dependent on the presence/absence and distribution of bacterial species but can be further understood through the independent and multifaceted investigation of bacterial metabolic, proteomic and gene expression profiles. This is demonstrated through the investigation of two phylogenetically distant bacterial groups, Lactobacilliales and Bifidobacteriales, that are commonly termed as 'lactic acid-producing bacteria'. The Lactobacillus genus is diverse, containing at least 12 separate phylogenetic groups with more than 150 species isolated from the mammalian GIT [13]. However, the microbiota (the assemblage of microorganisms present in a defined environment) consists of far more than just lactobacilli and bifidobacteria. The identification of these bacterial systems and the analysis of their contribution to host biochemistry and physiology has been accelerated through modern advanced analytical techniques that sit within 'omics' technologies and include metataxonomics, metatranscriptomics, (meta)proteomics and metabolomics/metabanomics [1] (Figure 3).

The human gut microbiome is genetically diverse, expressing approximately 150-times more genes than the human host [36]. Since it is agreed that the majority of the bacteria commonly identified in the microbiome cannot be routinely cultured using traditional laboratory techniques (ca. $80 \%$ or greater) [37], we rely on high-throughput sequencing approaches to gain a comprehensive insight into this complex microbial community [38]. Historically, the most common DNA-based approach applied to interrogate the microbiome applies targeted amplification of specific phylogenetic marker genes to make taxonomic inferences. This targeted approach is termed metataxonomics and is most frequently achieved through PCR amplification of regions of the ribosomal rRNA 16S gene, which are highly conserved across taxa [39]. Since this method only requires a sequence from a single gene, it represents a costeffective and widely utilized procedure for identifying species diversity in the gut microbiome. However, care should be exercised when targeting a precise region of the $16 \mathrm{~S}$ gene as this has been shown to have an influence on the type of bacteria queried and can introduce experimental bias [40]. Data outcomes resulting from this method of analysis are clustered into operational taxonomic units based on their similarity to other sequences in the community [41]. Taxonomic classifications are then assigned using reference databases such as the Ribosomal Database Project (RDP), Greengenes, Open Tree of Life Taxonomy (OTT) and SILVA NCBI to define taxonomy [42]. A metataxonomic approach was ubiquitously applied in the initial phase of HMP1, which characterized the microbiomes of healthy individuals across five anatomical locations [43].

Rather than amplifying a specific phylogenetic marker, metagenomics utilizes high-throughput sequencing to provide a non-targeted analysis of all DNA extracted [1]. DNA is first isolated, then randomly sheared using a shotgun approach, sequenced and analyzed to generate a full genomic profile of the microbiome [44]. Overlapping sequences of DNA are assembled into longer genomic sequence contigs that are subsequently annotated against 


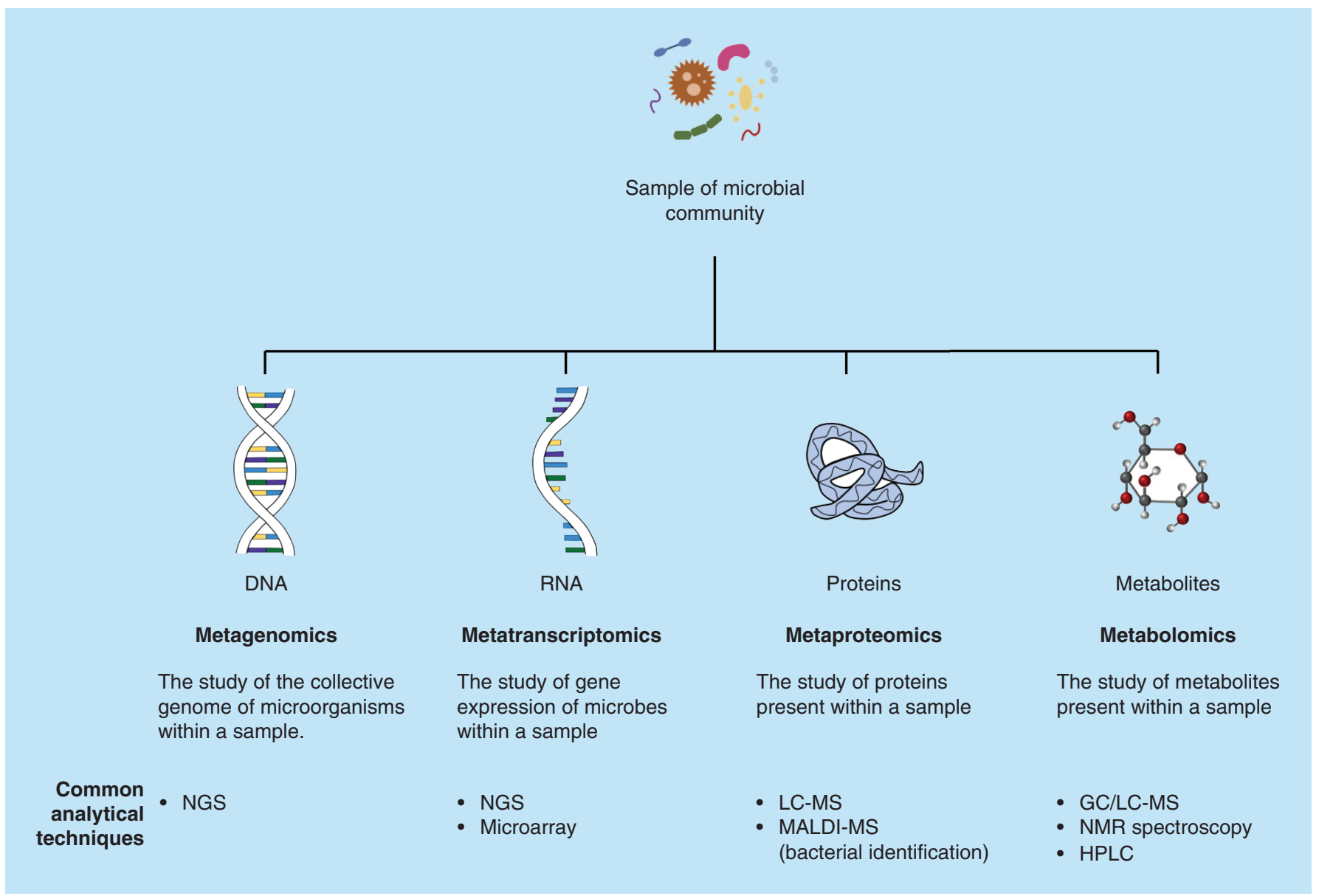

Figure 3. Overview of multi-omics technologies and associated analytical techniques.

GC: Gas chromatography; HPLC: High-performance liquid chromatography; LC-MS: Liquid chromatography-mass spectrometry; MALDI: Matrix-assisted laser desorption ionization; MS: Mass spectrometry; NGS: Next-generation sequencing; NMR = nuclear magnetic resonance.

existing databases using complex algorithms for phylogenetic and functional analysis, such as MG-RAST, IMG/M and EBI Metagenomics [45]. Metagenomics was first described in 1998 by Handelsman \& Rodon and has since provided an unparalleled insight into the functional genetic profiles of microbial communities [46]. The approach enables the analysis of fundamental microbial pathways associated with the gut microbiome, as well as genes associated with antibiotic resistance and the co-evolution between microbiota and host. To date, several sequencing platforms have been applied to metagenomics ranging from traditional Sanger sequencing to high-throughput nextgeneration sequencing using 454/Roche and Illumina/Solexa systems, with each method presenting advantages and disadvantages relating to insert size, read length, depth, sequence accuracy, usability and cost [47]. For a comprehensive and critical evaluation of these sequencing platforms we recommend the review by Kuczynski et al. [41].

The traditional genomic approaches previously described the profile of total cumulative DNA present in a sample. This, however, tells us little about epigenetic factors, such as which genes are expressed, which are suppressed and in what proportions. To answer these questions, we can apply metatranscriptomics. This approach analyses mRNA expression in the microbiome via the high-throughput sequencing of meta-cDNA [48]. By specifically analyzing genes that are actively expressed in the microbiome it becomes possible to compile a functional picture of metagenomic activity. Consequently, this form of analysis can provide adjunct information to existing metagenomics data and makes it a powerful tool for distinguishing dynamic changes in gene expression resulting from environmental factors that impact the microbiome, such as dietary changes [49]. Furthermore, outcomes from metatranscriptomic analyses have the capacity to distinguish co-evolutionary developments, which are important in enhancing our understanding of the symbiotic relationship between microbiome and host [50]. 
Increasingly, meta-genomic analyses are being combined with complementary approaches including metaproteomics and metabolomics/metabonomics to provide a meta-omics analysis that yields insights that cannot be obtained through a single method of analysis.

The biological system-wide collection of proteins carries the term proteome and was originally described as 'the total protein complement of a genome' [51]. This has led to an ever-increasing interest in the global study of proteins and their interactions, with the term proteomics described as 'the use of quantitative protein-level measurements of gene expression to characterize biological processes (e.g., disease processes and drug effects) and decipher the mechanisms of gene expression control' [52]. In contemporary discovery proteomics, also known as shotgun or bottom-up proteomics, proteins are cleaved into peptides using proteolytic enzymes and then analyzed by liquid chromatography coupled to high-resolution mass spectrometry [53]. This provides a peptide map of the analyzed sample that can then be stitched back together by specialist software to provide confident identification of measured proteins through the detection and measurement of known unique peptide fragments [53]. Subsequently, this affords the possibility of mapping the microbiota proteome, giving a functional overview of the proteins and polypeptides produced by the microbiota. In recent years, a substantial body of evidence has accumulated which suggests that certain surface-associated and extracellular products made by probiotic bacteria may be responsible for some of their mechanism of action. It is thought that these bacterial components interact directly with the host's mucosa and include: exopolysaccharides, bacteriocins, lipoteichoic acids and surface-associated and extracellular proteins [54].

The application of proteomics in probiotic research has mostly applied to the direct measurement of secreted or membrane proteins linked directly to the bacterial strains [55-57], with less emphasis on their interaction and subsequent impact on the total proteome of the host system - in other words, proteins present from the bacteriahost interaction. Nonetheless, attempts have been made to understand the proteomic changes following probiotic supplementation, with initial successes drawn from these analyses. For example, Reiff et al. [58] measured cecum proteins following a supplementation protocol of a commercial multistrain probiotic to IL-10 knockout mice, a model of inflammatory bowel disease (IBD), for 21 weeks. The data showed that 9 proteins increased and 2 decreased at a level greater than twofold, with a ninefold increase in measured LGALS2 that has previously been demonstrated in vitro to contribute to epithelial would healing and suppression of activated $T$ cells $[59,60]$. In addition, decreases in measurements of REG3G were seen in mice with decreased IBD severity following probiotic treatment compared with those which were administered a saline placebo. Similarly, in IBS an 8-week probiotic supplementation (Lactobacillus acidophilus NCFM and Bifidobacterium lactis HN019) to animals demonstrated that PPAR- $\gamma$ signaling proteins were maintained at levels equivalent to nonsupplemented healthy controls, compared with IBS-induced animals without supplementation that showed decreased PPAR- $\gamma$ content [61]. Conversely, it has also been demonstrated that probiotic supplementation exerts no impact on overall fecal proteome measurements and variances are more reflected through personalized differences in microbiome content [62].

Although there are limited investigations assessing the impact on the global metaproteome following probiotic supplementation, promising initial data point toward alterations in protein production that are related to health and disease and warrants further study.

Metabolomics refers to the study of metabolites produced and released through biochemical pathways that can be measured both systemically and at the cellular level and are an indication of the physiological state of the organism or system [63]. Furthermore, the term metabonomics describes the measurement of metabolites that are measured from a multi-organism system such as the human body and its incorporated microbiota [64,65]. The collection of metabolites produced by these organisms is collectively referred to as the metabolome [65]. Although microbiome-focused research has been performed on the global profiling of metabolites [66-68], the targeted analysis of gut-mediated metabolites has received more research interest over recent years. For example, gut bacterial metabolites have been shown to have mechanistic and associative effects on human health, with perhaps the most well-documented being the gut-mediated metabolite of trimethylamine $\mathrm{N}$-oxide, or more commonly known as TMAO. TMAO is produced through gut bacterial metabolism of dietary-derived quaternary amine molecules such as choline and L-carnitine [69,70]. Some bacteria possess the genetic code to cleave the trimethylamine (TMA) group from these molecules and release it as a metabolic by-product, with $\mathrm{Cut} C$ and $\mathrm{CntAB}$ the most common genes required for the digestion of choline and L-carnitine, respectively [71]. The released TMA is then absorbed through the gut wall into the portal vein and transported to the liver where it is converted by flavin-containing monooxygenases (e.g., FMO3) through $\mathrm{N}$-oxidation to TMAO [72] (Figure 4). 


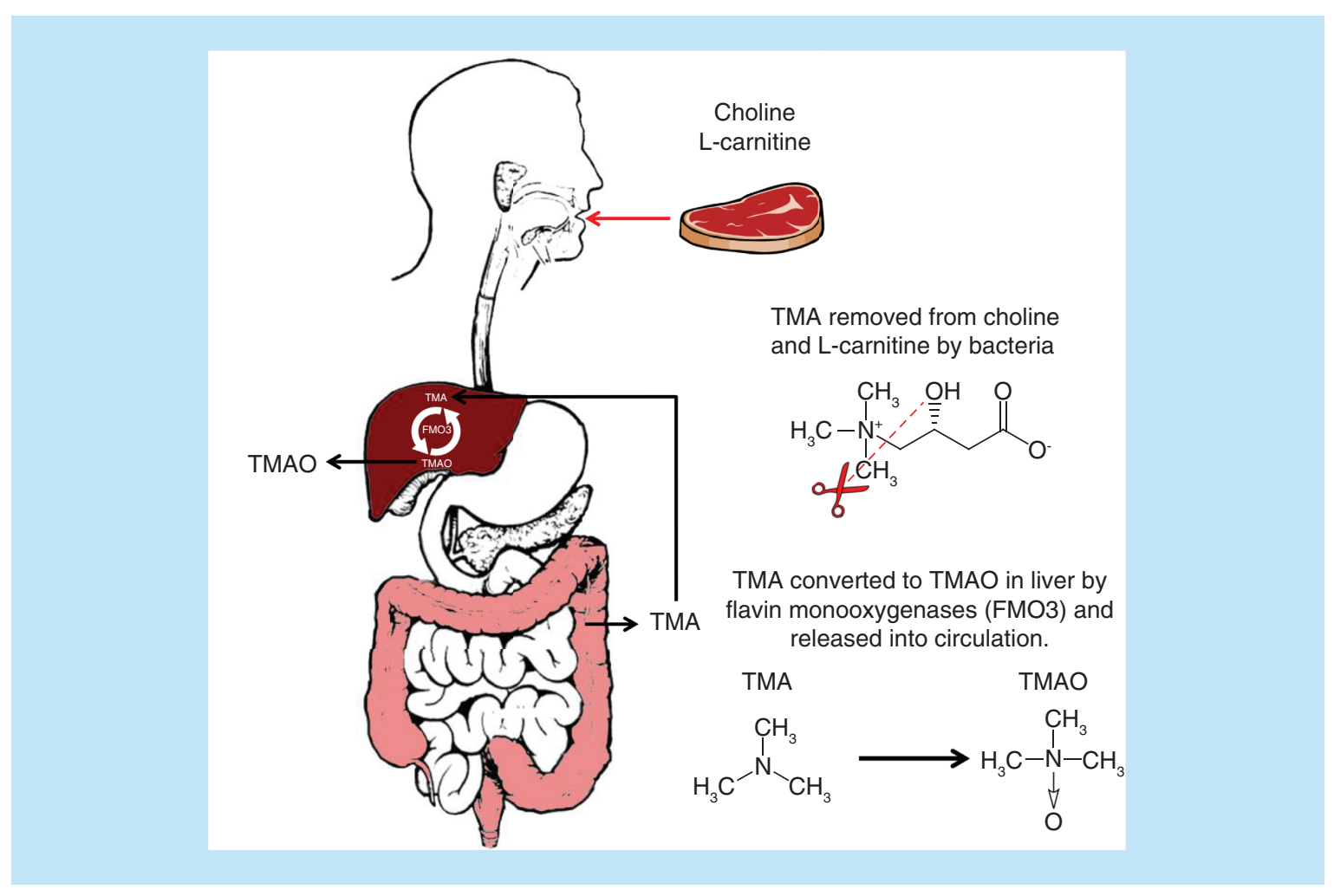

Figure 4. A schematic drawing to demonstrate gut bacterial metabolism of dietary components choline and L-carnitine into TMA which is subsequently converted to TMAO by liver flavin-containing monooxygenases (e.g., FMO3). TMA: Trimethylamine; TMAO: Trimethylamine N-oxide.

TMAO has gained worldwide media coverage through its associations with multiple diseases, centered primarily on cardiovascular disease. Elevated levels of TMAO have shown mechanistic effects in animal models through acceleration of atherosclerotic plaque formation [70], worsening of pressure overload-induced heart failure [73] and elevated fibrotic potential in renal tissue [74]. In addition to this, associations with increased circulating levels of TMAO (measured in plasma and serum) have been demonstrated as biomarkers able to independently predict the risk of adverse outcome in chronic and acute cardiac disease patients [75-78]. To this end, it is not surprising that there have been efforts to reduce the accumulation of TMAO within the systemic circulation, with chemical inhibitors of TMA-lyase activity shown to effectively reduce bacterial production of TMA [79,80]. This chemical approach has yet to be translated to human patient cohorts, but efforts to reduce TMA/TMAO levels through probiotic supplementation have been made albeit with mixed success [81-85]. The dosing of human participants with commercially available probiotic products is unlikely to have a meaningful effect on TMAO production. A more focused analysis of the mechanistic actions of particular bacterial strains and their metabolic capabilities is required before any health claim benefits can be realized.

Potential probiotic (and prebiotic) approaches have further been identified with the view to the increased production of certain metabolite by-products. For example, short-chain fatty acids (SCFAs, particularly acetate, propionate and butyrate) are predominantly produced following the fermentation of dietary fiber and have shown positive associations with the reduction in pathophysiological processes of disease [86]. Of note, Andrade-Oliveira et al. [87] demonstrated that increased bioavailability of fecal and circulating acetate could be achieved through probiotic supplementation of known acetate-producers Bifidobacterium adolescentis or B. longum. Subsequent experiments in mice showed that the probiotic supplement ameliorated ischemia-induced renal tissue damage to a similar degree as to when acetate was administered by chemical supplementation $(200 \mathrm{mg} / \mathrm{kg}$ acetic acid in PBS buffered to $\mathrm{pH}$ 7.4). Further, colocalization of the probiotic treatment was confirmed by the detection of fluorescently labeled bacteria in the intestinal niche, albeit at a low occurrence. A high-fiber diet (which functions as a prebiotic approach to increase SCFA production [88]) has also achieved positive therapeutic effects in kidney 
disease, with improvements in renal function and blood pressure coupled with decreased levels of inflammation, oxidative stress and fibrosis [89].

Altogether, the impact of gut-mediated metabolites on the human biological system provides promising applications of probiotic supplementation combined with nutritional strategies to modulate bacterial metabolite production and subsequently improve health and disease characteristics.

In isolation, all of the described omics-based investigations offer an abundance of data and potential information on the workings of the microbiome to further characterize the complex nature of the microbiota-host interaction. Increasingly, meta-genetic analyses are being combined with complementary approaches including metaproteomics and metabolomics to provide a meta-omic analysis that yields insights that cannot be obtained through a single method of analysis. The ability to combine these datasets to generate meta-omics investigations will provide an improved understanding of the elaborate processes in place to elucidate the underlying biological basis and mechanism of action of probiotics.

\section{The biological basis \& mechanisms of action of probiotics}

The heterogeneous nature of the gut microbiota is mirrored in the varied mechanisms of action of different microbial species. The biological effects of probiotics can be divided into several broad categories:

- Pathogen resistance: Probiotics are thought to maintain, or re-establish, host microbial homeostasis and correspondingly reduce pathogenic invasion and colonization. If endogenous microbes are able to occupy all functional niches, there is a reduced likelihood of pathogenic invasion and colonization in that ecosystem. Probiotics can either occupy functional niches that are left open by the endogenous community (preventing opportunistic infection) or they may directly alter the local environment through the secretion of SCFAs, lactic acid, bacteriocins and reactive oxygen species to inhibit the growth of pathogenic organisms [90].

- Nutritional functionality: Certain species of the gut microbiota contribute to vitamin availability and production of SCFAs. Vitamin K, vitamin B12, pyridoxine, biotin, folate, nicotinic acid and thiamine can all be produced by gut micro-organisms [11]. Furthermore, butyrate is a major energy source for enterocytes and is involved in the maintenance of the enteric mucosa [92].

- Immune functionality: Probiotics have been shown to have diverse effects on the immune system. Some probiotic species can be categorized as immunostimulatory (i.e., pro-inflammatory) and are characterized by their ability to induce IL-12 and natural killer (NK) cell immunity [93]. Other species are categorized as immunoregulatory (i.e., anti-inflammatory) due to their ability to induce IL-10 and regulatory T cell pathways [94]. The overall effect on the immune system is totally dependent upon the specific probiotic species or strain, with some acting as pro-inflammatory and others as anti-inflammatory agents.

- Amelioration of contaminants: Certain probiotics can lower the risk from ingested hazardous compounds. For example, Pedicococcus pentosaecus breaks down fumonisins, a group of mycotoxins produced from fungi, which are found on a wide variety of crops [91].

- Xenobiotic and drug metabolism: Recent research has shown that the gut microbial metabolite $p$-cresol can reduce the capacity of the liver to metabolize paracetamol due to competitive inhibition of hepatic sulfotransferases. The role of the gut microbiota in drug and xenobiotic metabolism could have a profound impact on therapy options for various conditions in the future [95]. Furthermore, there is increasing evidence to show that dietary and environmental chemical pollutants can interfere with gut bacterial function (transcription, metabolism etc.) and can consequently affect host health through inducing a pro-inflammatory response within the gut $[96,97]$.

- Bile acid metabolism: Certain species of the gut microbiota - such as Bacteroides intestinalis - have been shown to deconjugate and dehydrate primary bile acids to convert them into secondary bile acids [95]. Secondary bile acids can inhibit Clostridium difficile spore germination and, therefore, suppress the vegetative growth of $C$. difficile [98].

\section{Clinical probiotic research}

Probiotics have been extensively studied in gastrointestinal diseases including: infectious gastroenteritis, antibioticassociated diarrhea (including CDAD), IBD and IBS $[15,23,99,100]$. A 2017 Cochrane review of 31 trials in the prevention of $\mathrm{CDAD}$ concluded that probiotics achieved a risk reduction of $60 \%$ (number needed to treat to benefit one person $=42$ patients, 95\% CI: 32-58) [16]. Based on this review, probiotics have been recommended as 'safe and effective when used in conjunction with antibiotics for the treatment of $C$. difficile' in the National Institute for Health and Care Excellence (NICE) Clinical Knowledge Summaries webpage [101]; a service that 
provides primary care practitioners with summaries of the current evidence base and practical guidance on best practice in many common presentations. However, a 2018 review of Cochrane reviews concluded that probiotics, in the management of IBD (both Crohn's and ulcerative colitis), lack sufficient evidence to allow their benefits to be determined and that more research is required [99]. Numerous probiotic products have been trialed in the management of IBS, as described earlier, and the 2017 NICE clinical practice guidelines for IBS in adults found good-quality evidence that combination (multi-strain) probiotics significantly improve global IBS symptoms, pain, and bloating compared with placebo [102]. In support of this, the largest probiotic RCT to date in IBS (400 patients in a randomized approach) found that a 14-strain probiotic significantly improved all these symptoms, and also substantially increased quality of life [103].

Obesity, and its related metabolic diseases, are a burgeoning international problem for both health and healthcare systems. The combined medical costs associated with treating obesity-related diseases are estimated to increase by \$48-66 billion per annum in the USA and by $£ 1.9-2.0$ billion per annum in the UK by 2030 [104]. Current management of obesity with long-term medication or surgical intervention have been shown to be efficacious but not without considerable side effects $[105,106]$. Probiotics, as an adjunct to healthy diet and lifestyle, may offer a low-risk option for such patients. In a 2018 review of 15 probiotic RCTs in overweight or obese subjects, probiotics achieved a significantly larger reduction in body weight and BMI compared with placebo; albeit with small effect sizes [107]. Intriguingly, in the largest subsequent RCT to date, researchers identified that a heat-killed probiotic strain (Bifidobacterium animalis subsp. lactis CECT 8145) lead to the most significant reductions in waist circumference and waist to height ratio compared with placebo, thus suggesting that the beneficial effects might be obtained from nonviable bacterial components [108].

The relationship between the gut microbiota and neuropsychiatric conditions is also proving to be a rapidly evolving field of research. Referred to as the 'gut-brain axis,' this describes the bidirectional communication system that exists between the GIT and the central nervous system, utilizing neurological, immunological and hormonal signaling pathways [109]. Promising preclinical research has demonstrated that probiotic supplementation can alter the gut microbiota to ameliorate anxiety-like behavior [18] and depressive-like behavior in mice [110]. Several further rodent studies have shown that consumption of probiotics can prevent increases in certain stress hormones, including ACTH, corticosterone and epinephrine via the hypothalamic-pituitary-adrenal axis [19]. Human clinical trials examining the effect of probiotics on neuropsychiatric conditions are relatively small in number at present but a recent systematic review provides an interesting perspective on the current evidence base for the effects of probiotics on symptoms of depression and anxiety [19]. The clinical trials to date demonstrate an exciting - if somewhat heterogenous - set of results that in many ways mirror other areas of probiotic research. In a 2016 randomized placebo-controlled trial, Akkasheh et al. [111] demonstrated a significant reduction in depressive symptom scores (as measured by the Beck's Depression Inventory) following an 8-week period of probiotic supplementation with Lactobacillus acidophilus, L. casei and Bifidobacterium bifidum. Furthermore, Rao and colleagues [112] conducted a randomized placebo-controlled trial examining the effect of $L$. casei on anxiety symptoms in patients with chronic fatigue syndrome. In this trial, it was observed that consumption of $L$. casei daily for 2 months led to a significant decrease in anxiety symptoms among those taking the probiotic when compared with controls, further adding to the evidence base. In a recent review, Romijn \& Rucklidge [113] coined the term 'psychobiotics' to refer to probiotics which produce a health benefit in patients suffering from psychiatric illness.

However, psychobiotics are not the only focus of probiotic research into the gut-brain axis. Epidemiological data demonstrate an interesting correlation between migraine sufferers and gastrointestinal conditions, such as coeliac disease and IBD [114]. In light of this, researchers investigated the effects of a multispecies probiotic supplement on migraine patients [115]. The trial included individuals diagnosed with either episodic or chronic migraine (as defined by the International Headache Society). Measured against placebo controls, individuals that received the multispecies probiotic experienced a statistically and clinically significant reduction in frequency and severity of migraine attacks, in addition to a reduction in weekly abortive drug usage. While the pathophysiology of migraine is phenomenally complex and yet to be definitively characterized [116], this trial adds to the evidence base that migraine is a multisystem condition, and provides a novel potential avenue for migraine prophylaxis.

Although gastrointestinal diseases have been the traditional focus in microbiome and probiotic research, the scope has been dramatically broadened in recent years to include virtually every organ system in the body. Probiotics have demonstrated efficacy in reducing blood urea levels in chronic renal failure [117], treating and preventing atopic dermatitis [118,119], reducing transaminases and measures of fibrosis in non-alcoholic steatohepatitis [120], improving dysphoria and depression [19], preventing antibiotic-associated diarrhea [15] and even treating symptoms 
of ophthalmological conditions such as vernal keratoconjunctivitis [121]. The research in many of these areas is in its infancy, and there is a requirement to better understand the underlying mechanisms of action, in conjunction with high-quality Phase III studies, if probiotics are to be integrated into widespread mainstream clinical practice.

\section{Conclusion}

The probiotics industry is an ever-growing entity with continual expansion of products being taken to market. This has driven scientific research with the aspirations to uncover probiotic strains that provide conclusive evidence of improvements in health and disease outcomes. These opportunistic endpoints have not currently been met, evidenced by the fact that no certified health claims credited to probiotic products are currently in place. This is likely owing to the wide inter-personal variations in commensal bacteria as well as fundamental differences between probiotic strains. The further application of advanced omics technologies will provide an improved understanding of the complex host-bacteria interactions, but research strategies must be well planned to provide informative data that can be biologically interpreted. Once these biological mechanisms and physiological interactions can be elucidated and more confidently defined, the translation of laboratory science to clinical treatments using probiotics supplements will be substantially accelerated.

Albeit currently at an early stage, the future for probiotic research provides the exciting potential to influence conditions related to both health and disease.

\section{Future perspective}

Although a wealth of literature on probiotic research is available, many of these relate to high-throughput analyses of the gut microbiota that provide snapshots of the diversity at play in the microbiome, however this yields little information about how this complex system interacts. Research efforts in the coming years are likely to focus on the generation and combining of data from multiple omics platforms. This will allow in-depth characterization of the microbiome through its genetic make-up and transcription products (metagenomics/metatranscriptomics), to its proteins (metaproteomics) and metabolic products (metabolomics/metabonomics). Integrating these meta-omics datasets to create a systems level framework that links the data through a comprehensive mechanistic model of the microbiome will be one of the greatest future challenges of microbiome research [122]. However, through the combination of these multiple technologies it will be possible to unlock the workings of this complex ecosystem and understand which microbial components are functionally relevant. Once this is better understood then it will be possible to harness individual strains for their specific health benefits with the ultimate aim of creating

\section{Executive Summary}

- Lots of research, but still no health claim: insufficient evidence from large-scale studies has led to the inability to satisfy stringent regulations to legally associate a health claim to a probiotic supplement.

- Diversity, dysbiosis and strain specificity: The Human Microbiome Project has demonstrated the extensive complexity in commensal bacteria residing within human hosts. This wide genetic diversity between probiotic strains, coupled with the inter-personal variations in microbiome content, mean that the ability to define a 'healthy' or 'dysbiotic' microbiota is not without challenges that are dependent on the specific scenario being investigated.

- Multi-omics technologies to revolutionize and accelerate microbiome science: advanced analytical technologies have already begun to revolutionize the study of the microbiome, with individual omics technologies providing insightful data in the workings and interactions of the microbiota. Improved connectivity of these technologies will provide an increased ability to delineate the physiological and biochemical processes of host-bacteria interactions to accelerate future potential for applications in health and disease.

- The biological basis and mechanisms of action of probiotics: with diverse biological functions and mechanisms apparent across different bacterial strains, it is important for researchers and probiotic product developers to understand the properties of each strain and apply these proactively to target a preferred physiological interaction/response. For example, it is likely to be important and beneficial for probiotic strains that exhibit anti-inflammatory properties to be applied to research in health conditions associated with an augmented inflammatory response (e.g., Crohn's disease).

- Clinical probiotic research: An early and consistent research focus on gastrointestinal diseases is now resulting in inclusion of probiotics into evidence-based guidance for clinicians. Beyond the gut, the scope of clinical conditions amenable to probiotic management seems almost limitless. However, although the results from research in new indications, such as neurological pathologies, are very promising, a substantial amount of further work is required to provide healthcare providers with the confidence to embrace probiotics into regular practice. 
individualized probiotic treatments for specific indications and diagnoses and apply these to robust RCTs for clinical benefit.

\section{Author contributions}

All authors contributed to reviewing the literature and writing the manuscript.

\section{Financial \& competing interests disclosure}

RLJ Day and AJ Harper are employees of ADM Protexin Ltd. and RM Woods is funded through a Yakult Honsha Co., Ltd research grant. The authors have no other relevant affiliations or financial involvement with any organization or entity with a financial interest in or financial conflict with the subject matter or materials discussed in the manuscript apart from those disclosed.

No writing assistance was utilized in the production of this manuscript.

\section{Open access}

This work is licensed under the Attribution-NonCommercial-NoDerivatives 4.0 Unported License. To view a copy of this license, visit http://creativecommons.org/licenses/by-nc-nd/4.0/

\section{References}

Papers of special note have been highlighted as: $\bullet$ of interest; $\bullet \bullet$ of considerable interest

1. Marchesi JR, Ravel J. The vocabulary of microbiome research: a proposal. Microbiome 3(1), 31 (2015).

-. Provides an interesting resource for the description of language and terminology used in microbiome research.

2. Thaiss CA, Zmora N, Levy M, Elinav E. The microbiome and innate immunity. Nature 535(7610), 65-74 (2016).

3. Azcarate-Peril MA, Ritter AJ, Savaiano D et al. Impact of short-chain galactooligosaccharides on the gut microbiome of lactose-intolerant individuals. Proc. Natl Acad. Sci. USA 114(3), E367-E375 (2017).

4. Fetissov SO. Role of the gut microbiota in host appetite control: bacterial growth to animal feeding behaviour. Nat. Rev. Endocrinol. 13(1), 11-25 (2017).

5. van de Wouw M, Schellekens H, Dinan TG, Cryan JF. Microbiota-gut-brain axis: modulator of host metabolism and appetite. J. Nutr. 147(5), 727-745 (2017).

6. Jiang H, Ling Z, Zhang Y et al. Altered fecal microbiota composition in patients with major depressive disorder. Brain Behav. Immun. 48, 186-194 (2015).

7. Miele L, Giorgio V, Alberelli MA, De Candia E, Gasbarrini A, Grieco A. Impact of gut microbiota on obesity, diabetes, and cardiovascular disease risk. Curr. Cardiol. Rep. 17(12), 120 (2015).

8. Aron-Wisnewsky J, Clément K. The gut microbiome, diet, and links to cardiometabolic and chronic disorders. Nat. Rev. Nephrol. 12(3), 169-181 (2016).

9. Leung C, Rivera L, Furness JB, Angus PW. The role of the gut microbiota in NAFLD. Nat. Rev. Gastroenterol. Hepatol. 13(7), 412-425 (2016).

10. Budden KF, Gellatly SL, Wood DLA et al. Emerging pathogenic links between microbiota and the gut-lung axis. Nat. Rev. Microbiol. 15(1), 55-63 (2017).

11. Wilson Tang WH, Hazen SL. The gut microbiome and its role in cardiovascular diseases. Circulation. 135(11), 1008-1010 (2017).

12. Yu L-X, Schwabe RF. The gut microbiome and liver cancer: mechanisms and clinical translation. Nat. Rev. Gastroenterol. Hepatol. 14(9), 527-539 (2017).

13. Schirmer M, Franzosa EA, Lloyd-Price J et al. Dynamics of metatranscription in the inflammatory bowel disease gut microbiome. Nat. Microbiol. 3(3), 337-346 (2018).

14. van den Nieuwboer M, van de Burgwal LHM, Claassen E. A quantitative key-opinion-leader analysis of innovation barriers in probiotic research and development: valorisation and improving the tech transfer cycle. PharmaNutrition. 4(1), 9-18 (2016).

15. Chamberlain R, Lau C. Probiotics are effective at preventing Clostridium difficile-associated diarrhea: a systematic review and meta-analysis. Int. J. Gen. Med. 9, 27 (2016).

16. Goldenberg JZ, Yap C, Lytvyn L et al. Probiotics for the prevention of Clostridium difficile-associated diarrhea in adults and children. Cochrane Database Syst. Rev. 12, CD006095 (2017).

17. Notay M, Foolad N, Vaughn AR, Sivamani RK. Probiotics, Prebiotics, and synbiotics for the treatment and prevention of adult dermatological diseases. Am. J. Clin. Dermatol. 18(6), 721-732 (2017).

18. Bercik P, Park AJ, Sinclair D et al. The anxiolytic effect of Bifidobacterium longum NCC3001 involves vagal pathways for gut-brain communication. Neurogastroenterol. Motil. 23(12), 1132-1139 (2011).

19. Wallace CJK, Milev R. The effects of probiotics on depressive symptoms in humans: a systematic review. Ann. Gen. Psychiatry. 16(1), 14 (2017). 
20. Liang S, Wu X, Hu X, Wang T, Jin F. Recognizing depression from the microbiota-gut-brain axis. Int. J. Mol. Sci. 19(6), 1592 (2018).

21. Binnendijk KH, Rijkers GT. What is a health benefit? An evaluation of EFSA opinions on health benefits with reference to probiotics. Benef. Microbes. 4(3), 223-230 (2013).

22. Rijkers GT, de Vos WM, Brummer R-J, Morelli L, Corthier G, Marteau P. Health benefits and health claims of probiotics: bridging science and marketing. Br. J. Nutr. 106(09), 1291-1296 (2011).

23. McKenzie YA, Thompson J, Gulia P, Lomer MCE. British Dietetic Association systematic review of systematic reviews and evidence-based practice guidelines for the use of probiotics in the management of irritable bowel syndrome in adults (2016 update). J. Hum. Nutr. Diet. 29(5), 576-592 (2016).

24. Morgan XC, Segata N, Huttenhower C. Biodiversity and functional genomics in the human microbiome. Trends Genet. 29(1), 51-58 (2013).

25. Sender R, Fuchs S, Milo R. Revised estimates for the number of human and bacteria cells in the body. PLoS Biol. 14(8), 1-14 (2016).

26. Turroni S, Rampelli S, Centanni M et al. Enterocyte-associated microbiome of the hadza hunter-gatherers. Front. Microbiol. 7(3654), $1-12(2016)$

27. Clemente JC, Pehrsson EC, Blaser MJ et al. The microbiome of uncontacted Amerindians. Sci. Adv. 1(3), e1500183 (2015).

28. Moeller AH. The shrinking human gut microbiome. Curr. Opin. Microbiol. 38, 30-35 (2017).

29. Mosca A, Leclerc M, Hugot JP. Gut microbiota diversity and human diseases: should we reintroduce key predators in our ecosystem? Front. Microbiol. 7, 455 (2016).

30. Charbonneau MR, Blanton LV, DiGiulio DB et al. A microbial perspective of human developmental biology. Nature 535(7610), 48-55 (2016).

31. Hooks KB, O’Malley MA. Dysbiosis and its discontents. MBio 8(5), 1-11 (2017).

32. Lloyd-Price J, Abu-Ali G, Huttenhower C. The healthy human microbiome. Genome Med. 8(1), 51 (2016).

33. Turnbaugh PJ, Hamady M, Yatsunenko T et al. A core gut microbiome in obese and lean twins. Nature 457(7228), 480-484 (2009).

34. Azaïs-Braesco V, Bresson JL, Guarner F, Corthier G. Not all lactic acid bacteria are probiotics, ... but some are. Br. J. Nutr. 103(07), 1079 (2010).

35. Douillard FP, Ribbera A, Kant $\mathrm{R}$ et al. Comparative genomic and functional analysis of 100 lactobacillus rhamnosus strains and their comparison with strain GG. PLoS Genet. 9(8), e1003683 (2013).

- Demonstrates the vast genomic and functional differences observed between different strains of a single species.

36. Qin J, Li R, Raes J et al. A human gut microbial gene catalogue established by metagenomic sequencing. Nature 464(7285), 59-65 (2010).

37. Browne HP, Forster SC, Anonye BO et al. Culturing of 'unculturable' human microbiota reveals novel taxa and extensive sporulation. Nature 533(7604), 543-546 (2016).

38. Nichols D, Cahoon N, Trakhtenberg EM et al. Use of Ichip for high-throughput in situ cultivation of "uncultivable" microbial species. Appl. Environ. Microbiol. 76(8), 2445-2450 (2010).

39. Ziesemer KA, Mann AE, Sankaranarayanan $\mathrm{K}$ et al. Intrinsic challenges in ancient microbiome reconstruction using $16 \mathrm{~S}$ rRNA gene amplification. Sci. Rep. 5(1), 16498 (2015).

40. Kim D, Hofstaedter CE, Zhao C et al. Optimizing methods and dodging pitfalls in microbiome research. Microbiome 5(1), 52 (2017).

41. Kuczynski J, Lauber CL, Walters WA et al. Experimental and analytical tools for studying the human microbiome. Nat. Rev. Genet. 13(1), 47-58 (2012).

-. This is a comprehensive review of the analytical methods employed in genetic analyses of the gut microbiome.

42. Balvočiūtè M, Huson DH. SILVA, RDP, Greengenes, NCBI and OTT - how do these taxonomies compare? BMC Genomics 18(S2), 114 (2017)

43. Huse SM, Ye Y, Zhou Y, Fodor AA. A Core Human Microbiome as Viewed through 16S rRNA Sequence Clusters. PLoS ONE 7(6), e34242 (2012).

44. Quince C, Walker AW, Simpson JT, Loman NJ, Segata N. Shotgun metagenomics, from sampling to analysis. Nat. Biotechnol. 35(9), 833-844 (2017).

45. Mitchell AL, Scheremetjew M, Denise H et al. EBI Metagenomics in 2017: enriching the analysis of microbial communities, from sequence reads to assemblies. Nucleic Acids Res. 46(D1), D726-D735 (2018).

46. Handelsman J, Rondon MR, Brady SF, Clardy J, Goodman RM. Molecular biological access to the chemistry of unknown soil microbes: a new frontier for natural products. Chem. Biol. 5(10), R245-R249 (1998).

47. Thomas T, Gilbert J, Meyer F. Metagenomics - a guide from sampling to data analysis. Microb. Inform. Exp. 2(1), 3 (2012).

48. Gosalbes MJ, Durbán A, Pignatelli M et al. Metatranscriptomic approach to analyze the functional human gut microbiota. PLoS ONE 6(3), e17447 (2011). 
49. Franzosa EA, Morgan XC, Segata N et al. Relating the metatranscriptome and metagenome of the human gut. Proc. Natl Acad. Sci. USA 111(22), E2329-E2338 (2014).

50. Aguiar-Pulido V, Huang W, Suarez-Ulloa V, Cickovski T, Mathee K, Narasimhan G. Metagenomics, metatranscriptomics, and metabolomics approaches for microbiome analysis. Evol. Bioinforma. 12(Suppl. 1), 5-16 (2016).

51. Wasinger VC, Cordwell SJ, Cerpa-Poljak A et al. Progress with gene-product mapping of the Mollicutes: Mycoplasma genitalium. Electrophoresis 16(1), 1090-1094 (1995).

52. Anderson NL, Anderson NG. Proteome and proteomics: New technologies, new concepts, and new words. Electrophoresis 19(11), 1853-1861 (1998).

53. Aebersold R, Mann M. Mass spectrometry-based proteomics. Nature. 422(6928), 198-207 (2003).

54. Vinusha KS, Deepika K, Johnson TS, Agrawal GK, Rakwal R. Proteomic studies on lactic acid bacteria: a review. Biochem. Biophys. Reports. 14, 140-148 (2018).

55. Majumder A, Sultan A, Jersie-Christensen RR et al. Proteome reference map of Lactobacillus acidophilus NCFM and quantitative proteomics towards understanding the prebiotic action of lactitol. Proteomics. 11(17), 3470-3481 (2011).

56. Siciliano RA, Mazzeo MF. Molecular mechanisms of probiotic action: a proteomic perspective. Curr. Opin. Microbiol. 15(3), 390-396 (2012).

57. De Angelis M, Calasso M, Cavallo N, Di Cagno R, Gobbetti M. Functional proteomics within the genus Lactobacillus. Proteomics. 16(6), 946-962 (2016).

58. Reiff C, Delday M, Rucklidge G et al. Balancing inflammatory, lipid, and xenobiotic signaling pathways by VSL\#3, a biotherapeutic agent, in the treatment of inflammatory bowel disease. Inflamm. Bowel Dis. 15(11), 1721-1736 (2009).

59. Paclik D, Lohse K, Wiedenmann B, Dignass AU, Sturm A. Galectin-2 and -4, but not Galectin-1, promote intestinal epithelial wound healing in vitro through a TGF-beta-independent mechanism. Inflamm. Bowel Dis. 14(10), 1366-1372 (2008).

60. Paclik D, Berndt U, Guzy C et al. Galectin-2 induces apoptosis of lamina propria T lymphocytes and ameliorates acute and chronic experimental colitis in mice. J. Mol. Med. 86(12), 1395-1406 (2008).

61. Chen Q, Ren Y, Lu J et al. A novel prebiotic blend product prevents irritable bowel syndrome in mice by improving gut microbiota and modulating immune response. Nutrients 9(12), 1341 (2017).

62. Kolmeder CA, Salojärvi J, Ritari J et al. Faecal metaproteomic analysis reveals a personalized and stable functional microbiome and limited effects of a probiotic intervention in adults. PLoS ONE 11(4), e0153294 (2016).

63. Heaney LM, Deighton K, Suzuki T. Non-targeted metabolomics in sport and exercise science. J. Sports Sci. (2017). doi: 10.1080/02640414.2017.1305122

64. Nicholson JK, Lindon JC, Holmes E. "Metabonomics": understanding the metabolic responses of living systems to pathophysiological stimuli via multivariate statistical analysis of biological NMR spectroscopic data. Xenobiotica. 29(11), 1181-1189 (1999).

65. Nicholson JK, Wilson ID. Understanding "global" systems biology: metabonomics and the continuum of metabolism. Nat. Rev. Drug Discov. 2(8), 668-676 (2003).

66. Bisanz JE, Seney S, McMillan A et al. A systems biology approach investigating the effect of probiotics on the vaginal microbiome and host responses in a double blind, placebo-controlled clinical trial of post-menopausal women. PLoS One. 9(8), 1-10 (2014).

67. Calvani R, Brasili E, Praticò G et al. Application of NMR-based metabolomics to the study of gut microbiota in obesity. J. Clin. Gastroenterol. 48, S5-S7 (2014).

68. Chung H-J, Sim J-H, Min T-S, Choi H-K. Metabolomics and lipidomics approaches in the science of probiotics: a review. J. Med. Food. 21(11), 1086-1095 (2018).

69. Wang Z, Klipfell E, Bennett BJ et al. Gut flora metabolism of phosphatidylcholine promotes cardiovascular disease. Nature. 472(7341), 57-65 (2011).

- Describes the discovery of a gut-mediated metabolite where elevated circulating levels showed increased associations with cardiovascular disease.

70. Koeth RA, Wang Z, Levison BS et al. Intestinal microbiota metabolism of L-carnitine, a nutrient in red meat, promotes atherosclerosis. Nat. Med. 19(5), 576-585 (2013).

71. Jameson E, Doxey AC, Airs R, Purdy KJ, Murrell JC, Chen Y. Metagenomic data-mining reveals contrasting microbial populations responsible for trimethylamine formation in human gut and marine ecosystems. Microb. Genomics 2(9), e000080 (2016).

72. Bennett BJ, Vallim TQDA, Wang Z et al. Trimethylamine-N-Oxide, a metabolite associated with atherosclerosis, exhibits complex genetic and dietary regulation. Cell Metab. 17(1), 49-60 (2013).

73. Organ CL, Otsuka H, Bhushan S et al. Choline diet and its gut microbe-derived metabolite, trimethylamine N-oxide, exacerbate pressure overload-induced heart failure. Circ. Hear. Fail. 9(1), (2016).

74. Tang WHW, Wang Z, Kennedy DJ et al. Gut microbiota-dependent trimethylamine N-oxide (TMAO) pathway contributes to both development of renal insufficiency and mortality risk in chronic kidney disease. Circ. Res. 116(3), 448-455 (2015). 
75. Tang WHW, Wang Z, Levison BS et al. Intestinal microbial metabolism of phosphatidylcholine and cardiovascular risk. N. Engl. J. Med. 368(17), 1575-1584 (2013).

76. Tang WHW, Wang Z, Fan Y et al. Prognostic value of elevated levels of intestinal microbe-generated metabolite trimethylamine- $\mathrm{N}$-oxide in patients with heart failure: Refining the gut hypothesis. J. Am. Coll. Cardiol. 64(18), 1908-1914 (2014).

77. Suzuki T, Heaney LM, Bhandari SS, Jones DJL, Ng LL. Trimethylamine N-oxide and prognosis in acute heart failure. Heart 102(11), 841-848 (2016).

78. Suzuki T, Heaney LM, Jones DJL, Ng LL. Trimethylamine N-oxide and risk stratification after acute myocardial infarction. Clin. Chem. 63(1), 420-428 (2017).

79. Wang Z, Roberts AB, Buffa JA et al. Non-lethal inhibition of gut microbial trimethylamine production for the treatment of atherosclerosis. Cell. 163(7), 1585-1595 (2015).

80. Roberts AB, Gu X, Buffa JA et al. Development of a gut microbe-targeted nonlethal therapeutic to inhibit thrombosis potential. Nat. Med. 24(9), 1407-1147 (2018).

81. Boutagy NE, Neilson AP, Osterberg KL et al. Probiotic supplementation and trimethylamine-N-oxide production following a high-fat diet. Obesity. 23(12), 2357-2363 (2015).

82. Matsumoto M, Kitada Y, Shimomura Y, Naito Y. Bifidobacterium animalis subsp. lactis LKM512 reduces levels of intestinal trimethylamine produced by intestinal microbiota in healthy volunteers: A double-blind, placebo-controlled study. J. Funct. Foods. 36, 94-101 (2017).

83. Borges NA, Stenvinkel P, Bergman P et al. Effects of probiotic supplementation on trimethylamine-n-oxide plasma levels in hemodialysis patients: a pilot study. Probiotics Antimicrob. Proteins. (2018). Doi: 10.1007/s12602-018-9411-1.

84. de Faria Barros A, Borges NA, Nakao LS et al. Effects of probiotic supplementation on inflammatory biomarkers and uremic toxins in non-dialysis chronic kidney patients: A double-blind, randomized, placebo-controlled trial. J. Funct. Foods. 46, 378-383 (2018).

85. Qiu L, Tao X, Xiong H, Yu J, Wei H. Lactobacillus plantarum ZDY04 exhibits a strain-specific property of lowering TMAO via the modulation of gut microbiota in mice. Food Funct. 9(8), 4299-4309 (2018).

86. Tan J, McKenzie C, Potamitis M, Thorburn AN, Mackay CR, Macia L. The role of short-chain fatty acids in health and disease. Adv Immunol. 121, 91-119 (2014).

87. Andrade-Oliveira V, Amano MT, Correa-Costa M et al. Gut bacteria products prevent AKI induced by ischemia-reperfusion. J. Am. Soc. Nephrol. 26(8), 1877-1888 (2015).

-. Demonstrated the successful application of a target probiotic strategy to increase acetate production and subsequently improve renal function following induced injury.

88. Lewis JD, Abreu MT. Diet as a trigger or therapy for inflammatory bowel diseases. Gastroenterology 152(2), 398-414.e6 (2017).

89. Al Khodor S, Shatat IF. Gut microbiome and kidney disease: a bidirectional relationship. Pediatr. Nephrol. 32(6), 921-931 (2017).

90. Harper A, Naghibi M, Garcha D. The role of bacteria, probiotics and diet in irritable bowel syndrome. Foods. 7(2), 13 (2018).

91. Vandenplas Y, Huys G, Daube G. Probiotics: an update. J. Pediatr. (Rio. J). 91(1), 6-21 (2015).

92. Ríos-Covián D, Ruas-Madiedo P, Margolles A, Gueimonde M, de los Reyes-Gavilán CG, Salazar N. Intestinal short chain fatty acids and their link with diet and human health. Front. Microbiol. 7(2-3), 207-216 (2016).

93. Aziz N, Bonavida B. Activation of natural killer cells by probiotics. For. Immunopathol. Dis. Therap. 7(1-2), 41-55 (2016).

94. Azad MAK, Sarker M, Wan D. Immunomodulatory effects of probiotics on cytokine profiles. Biomed Res. Int. 2018, 8063647 (2018).

95. Jandhyala SM. Role of the normal gut microbiota. World J. Gastroenterol. 21(29), 8787 (2015).

96. Jin Y, Wu S, Zeng Z, Fu Z. Effects of environmental pollutants on gut microbiota. Environ. Pollut. 222, 1-9 (2017).

97. Defois $\mathrm{C}$, Ratel J, Garrait $\mathrm{G}$ et al. Food chemicals disrupt human gut microbiota activity and impact intestinal homeostasis as revealed by in vitro systems. Sci. Rep. 8(1), 11006 (2018).

98. Ridlon JM, Kang D-J, Hylemon PB. Bile salt biotransformations by human intestinal bacteria. J. Lipid Res. 47(2), 241-259 (2006).

99. Parker EA, Roy T, D’Adamo CR, Wieland LS. Probiotics and gastrointestinal conditions: An overview of evidence from the Cochrane Collaboration. Nutrition. 45(2018), 125-134.e11 (2018).

100. Vandenplas Y. Probiotics and prebiotics in infectious gastroenteritis. Best Pract. Res. Clin. Gastroenterol. 30(1), 49-53 (2016).

101. NICE. Diarrhoea - antibiotic associated [Internet]. (2018). https://cks.nice.org.uk/diarrhoea-antibiotic-associated\#!scenario.

102. NICE. Clinical practice guideline. Irritable bowel syndrome in adults: diagnosis and management of irritable bowel syndrome in primary care [Internet]. (2017) www.nice.org.uk/guidance/cg61/evidence/full-guidance-pdf-196701661.

103. Ishaque SM, Khosruzzaman SM, Ahmed DS, Sah MP. A randomized placebo-controlled clinical trial of a multi-strain probiotic formulation (Bio-Kult ${ }^{\circledR}$ ) in the management of diarrhea-predominant irritable bowel syndrome. BMC Gastroenterol. 18(1), 71 (2018).

104. Wang YC, McPherson K, Marsh T, Gortmaker SL, Brown M. Health and economic burden of the projected obesity trends in the USA and the UK. Lancet 378(9793), 815-825 (2011). 
105. Yanovski SZ, Yanovski JA. Long-term drug treatment for obesity. JAMA 311(1), 74-86 (2014).

106. Chang S, Stoll CRT, Song J, Varela JE, Eagon CJ, Colditz GA. The effectiveness and risks of bariatric surgery. JAMA Surg. 149(3), 275-287 (2014).

107. Borgeraas H, Johnson LK, Skattebu J, Hertel JK, Hjelmesaeth J. Effects of probiotics on body weight, body mass index, fat mass and fat percentage in subjects with overweight or obesity: a systematic review and meta-analysis of randomized controlled trials. Obes. Rev. 19(2), 219-232 (2018).

108. Pedret A, Valls RM, Calderón-Pérez L et al. Effects of daily consumption of the probiotic Bifidobacterium animalis subsp. lactis CECT 8145 on anthropometric adiposity biomarkers in abdominally obese subjects: a randomized controlled trial. Int. J. Obes. (2018). doi: 10.1038/s41366-018-0220-0.

109. van Hemert S, Breedveld AC, Rovers JMP et al. Migraine associated with gastrointestinal disorders: review of the literature and clinical implications. Front. Neurol. 5, 241 (2014).

110. Sun J, Wang F, Hu X et al. Clostridium butyricum attenuates chronic unpredictable mild stress-induced depressive-like behavior in mice via the gut-brain axis. J. Agric. Food Chem. 66(31), 8415-8421 (2018).

111. Akkasheh G, Kashani-Poor Z, Tajabadi-Ebrahimi M et al. Clinical and metabolic response to probiotic administration in patients with major depressive disorder: a randomized, double-blind, placebo-controlled trial. Nutrition. 32(3), 315-320 (2016).

112. Rao AV, Bested AC, Beaulne TM et al. A randomized, double-blind, placebo-controlled pilot study of a probiotic in emotional symptoms of chronic fatigue syndrome. Gut Pathog. 1(1), 6 (2009).

113. Romijn AR, Rucklidge JJ. Systematic review of evidence to support the theory of psychobiotics. Nutr. Rev. 73(10), 675-693 (2015).

114. Dai Y-J, Wang H-Y, Wang X-J, Kaye AD, Sun Y-H. Potential beneficial effects of probiotics on human migraine headache: a literature review. Pain Phys. 20(2), E251-E255 (2017).

115. Martami F, Togha M, Seifishahpar M et al. The effects of a multispecies probiotic supplement on inflammatory markers and episodic and chronic migraine characteristics: A randomized double-blind controlled trial. Cephalalgia (2019). Doi: 10.1177/0333102418820102.

116. Goadsby PJ, Holland PR, Martins-Oliveira M, Hoffmann J, Schankin C, Akerman S. Pathophysiology of migraine: a disorder of sensory processing. Physiol. Rev. 97(2), 553-622 (2017).

117. Miranda Alatriste PV, Urbina Arronte R, Gómez Espinosa CO, Espinosa Cuevas M de los Á. Effect of probiotics on human blood urea levels in patients with chronic renal failure. Nutr. Hosp. 29(3), 582-90 (2014).

118. Zhao M, Shen C, Ma L. Treatment efficacy of probiotics on atopic dermatitis, zooming in on infants: a systematic review and meta-analysis. Int. J. Dermatol. 57(6), 635-641 (2018).

119. Zuccotti G, Meneghin F, Aceti A et al. Probiotics for prevention of atopic diseases in infants: systematic review and meta-analysis. Allergy 70(11), 1356-1371 (2015).

120. Ferolla SM. Probiotics as a complementary therapeutic approach in nonalcoholic fatty liver disease. World J. Hepatol. 7(3), 559 (2015).

121. Iovieno A, Lambiase A, Sacchetti M, Stampachiacchiere B, Micera A, Bonini S. Preliminary evidence of the efficacy of probiotic eye-drop treatment in patients with vernal keratoconjunctivitis. Graefe's Arch. Clin. Exp. Ophthalmol. 246(3), 435-441 (2008).

122. Waldor MK, Tyson G, Borenstein E et al. Where Next for Microbiome Research? PLoS Biol. 13(1), e1002050 (2015).

- Offers an interesting and insightful discussion on the potential future avenues for research targeted at the gut microbiome. 
